\title{
Leaning against the Bubble: Central Bank Intervention in Walrasian Asset Markets
}

\author{
Chia-Lin Chang ${ }^{1,2,3, *(\mathbb{D})}$, Jukka Ilomäki ${ }^{4}$ and Hannu Laurila 4 \\ 1 Department of Applied Economics, National Chung Hsing University, Taichung 402, Taiwan \\ 2 Department of Finance, National Chung Hsing University, Taichung 402, Taiwan \\ 3 Department of Finance, Asia University, Taichung 41354, Taiwan \\ 4 Faculty of Management and Business, Tampere University, FI-33014 Tampere, Finland; \\ jukka.ilomaki@tuni.fi (J.I.); hannu.laurila@tuni.fi (H.L.) \\ * Correspondence: changchialin@email.nchu.edu.tw
}

Citation: Chang, Chia-Lin, Jukka Ilomäki, and Hannu Laurila. 2021. Leaning against the Bubble: Central Bank Intervention in Walrasian Asset Markets. Risks 9: 214. https://doi. org /10.3390/risks9120214

Academic Editor: Mogens Steffensen

Received: 12 October 2021

Accepted: 18 November 2021

Published: 1 December 2021

Publisher's Note: MDPI stays neutral with regard to jurisdictional claims in published maps and institutional affiliations.

Copyright: (C) 2021 by the authors. Licensee MDPI, Basel, Switzerland. This article is an open access article distributed under the terms and conditions of the Creative Commons Attribution (CC BY) license (https:/ / creativecommons.org/licenses/by/ $4.0 /)$.

\begin{abstract}
The paper presents a two-period Walrasian financial market model composed of informed and uninformed rational investors, and noise traders. The rational investors maximize second period consumption utility from the payoffs of trading risk-free holdings to risky assets in the first period. The central bank reacts directly to asset price movements by selling or buying assets to stabilize the market price. It is found that the intervention makes the risky asset's market price per share less sensitive to information shocks, which presses the market price towards its average price thus reducing price variance. The informed investors' prediction coefficient remains unaffected, but that of the uninformed investors is magnified, which cancels out the negative effect on shock sensitivity thus keeping the expected value of the risky asset's dividend constant. Finally, the introduction of the policy rule does not affect rational investors' risk per share. A general conclusion is that the central bank's policy can be regarded as an effective automatic stabilizer of financial markets.
\end{abstract}

Keywords: asymmetric information; CARA; expectations; Walrasian financial markets

JEL Classification: D82; E58; G11; G14; G32

\section{Introduction}

The intuition behind the standard "leaning against the wind", or "leaning against the bubble", as we call the policy herein, is that if there is evidence of a bubble in the financial market, the central bank can deflate the bubble by raising the market interest rate. Thus, in the case of overpricing, improving financial stability needs tightening of monetary policy.

In spite of its intuitively appealing idea, the practical advisability of the policy is controversial. For a long time, there was a widespread opinion that central banks should not intervene because of the difficulty in detecting the bubbles, and because the unintended costs may outweigh the intended benefits of the policy. The financial crisis of 2008 resulted in a more favorable attitude towards the policy, but debate on its usefulness is still ongoing (Ciccarone et al. 2020).

Dong et al. (2020) found that the efficacy of the leaning against the bubble policy depends on the nature of the shock and the type of the policy. They also noted that in some cases it is optimal to attach a negative reaction coefficient on asset price movements. This notion motivates the need to examine the efficacy of such policy, where the central bank directly intervenes in the financial market as a powerful market operator, selling and buying assets thus affecting the market price. This paper aims to respond to that need.

The paper is a theoretical analysis in the Walrasian framework, where perfect price mechanism makes simultaneously all markets clear. In the basic financial market model, there are rational investors and noise traders (see for example Campbell and Kyle 1993; Wang 1993). The rational investors include informed investors who have private information about the value of the risky asset, and uninformed investors who infer the value 
from the asset's market price. The Walrasian model is a bit problematic in the context of asymmetric information, because the model implies that information about market prices is transferred from informed to uninformed agents. Nevertheless, the framework has been regarded as most reasonable in that context, and it has been widely used in articles published in top journals since the 1970s (see e.g., Grossman and Stiglitz 1980; Admati 1985).

In our model, the central bank tackles bubbles by controlling asset prices in the market. This is done by altering the bank's own holdings of the assets. The policy rule reacts automatically to overpricing or underpricing by making the bank sell or buy risky assets in the market so that the price mechanism corrects any irrational deviations from the average asset value.

We investigate what happens, when the central bank enters the financial market with its reactive policy rule. The main findings are the following. The introduction of the central bank's policy reduces the sensitivity of the risky asset's market price to information shocks, which presses the market price towards its average thus reducing price variance. The informed investors' prediction coefficient remains unaffected, whereas that of the uninformed investors is magnified, which cancels out the fall in the sensitivity parameters so that the expected value of the risky asset's dividend remains unaltered. Upon receiving the market information, the rational investors' risk per share is not affected by the policy. The conclusion is that the central bank's market intervention makes the financial market more stable.

The remainder of the paper proceeds as follows. Section 2 gives a brief literature review, and Section 3 presents the basic model. Section 4 derives the financial market equilibrium, and Section 5 incorporates the central bank's policy rule into the model. Section 6 provides the results of the analyses, that is the implications of the implementation of the policy rule. Section 7 concludes and discusses the findings.

\section{Literature Review}

Early opponents of the leaning against the bubble policy include, for example, Bernanke and Gertler (2001), who argued that the central bank should only control inflation. Furthermore, Greenspan $(2002,2004)$ and Posen (2006), who argued that the central bank should focus only on inflation and macroeconomic stability, and that "cleaning up the mess" would be the proper action after a random financial market bubble has burst. White (2009) and Conlon (2015) deemed the policy ineffective because the fundamental value of risky assets is uncertain, and Brunnermeier and Schnabel (2016) referred to historical evidence concerning the difficulty in detecting stock market bubbles with confidence. Svensson (2017) showed that the costs of the policy exceed its benefits by a substantial margin.

The advocates of the policy include for example Bean (2004), Roubini (2006) and Yellen (2010), who highlighted the importance of central bank's activity in tackling market bubbles. Mishkin (2017) provided a review of studies concerning central banks' reactions to market bubbles after the 2007-2009 financial crisis and concluded that the central bank should intervene earlier rather than later, because the costs of cleaning up the mess policy might become very high.

In the paper by Mishkin (2017), leaning against the bubble policy is suitable for tackling credit-driven bubbles, while cleaning up is appropriate if the bubble inflates from irrational exuberance, meaning that investors' average expectations depart from rational expectations (Allen et al. 2006; Bacchetta and Van Wincoop 2008). In the behavioral finance literature (e.g., Shiller 1981; De Bondt and Thaler 1985), irrational exuberance has been connected to investors' bounded rationality. Hirshleifer (2015) provided a review of this literature.

Grossman and Stiglitz (1980) presented a two-period Walrasian financial market model, where trading leads to market equilibrium with fixed supply of assets. In their model, rational informed investors have noisy private information about the value of a risky asset, and rational uninformed investors infer the asset's value from the market price. In the two-period model of Hellwig (1980), rational investors with noisy private information allocate their wealth between a risk-free and a risky asset in the first period 
and consume the accumulated wealth in the second period. Admati (1985) included several risky assets in the model. Mendel and Shleifer (2012) elaborated the two-period model by including noise traders, assuming also that the informed investors receive another private signal in the second period.

Gali (2014) incorporated a standard leaning against the bubble policy into the analysis. In his overlapping generations model, the market price of the risky asset consists of fundamental value and rational bubble components, and the bubble is passed to the next generation developing with the risk-free interest rate. The conclusion was that the policy does not operate because the bubble component does not have a discount factor. Ilomäki and Laurila (2021) found that leaning against the bubble is effective if uninformed investors obey their "animal spirits" and discount their future anticipations. The Keynesian concept refers to instincts, proclivities and emotions, which bypass strict rationality in rapid decisions.

In his thorough cost-benefit analysis, Svensson (2017) showed that the costs of standard leaning against the bubble policy clearly exceed its benefits mainly due to the unintended effects of the policy. Therefore, it should be obvious that a more direct intervention in the financial market would cause fewer unintended consequences. A natural leaning against the bubble policy rule (or reaction function) would be to buy risky assets in exchange of riskless alternatives when the asset price is low and sell it when the price is high. We examine the effects of such a policy rule connected to market price movements around the average market price.

\section{The Basic Model}

The model builds on Grossman and Stiglitz (1980), Mendel and Shleifer (2012), and Ilomäki and Laurila $(2018,2020)$. In the two-period Walrasian financial market model, there is a set $[0,1]$ of rational constant absolute risk-averse investors, divided into informed investors with the share $\mu$ and uninformed investors with the share $1-\mu$. Both allocate their investments between risk-free and risky assets in the first period to maximize their consumption utility from the investment payoffs in the second period.

The constant absolute risk aversion (CARA) exponential utility function $u(c)=-e^{-c}$ is applied with a constant absolute risk aversion coefficient equal to one. The numeraire risk-free asset pays one unit of consumption in the second period, and the risky asset pays a random dividend $\widetilde{D} \sim N\left(\bar{D}, \sigma_{D}^{2}\right)$ in terms of consumption units in the second period. The market price of the risky asset is $P$ per share, expressed in terms of consumption.

The informed investors observe a noisy private signal: $\widetilde{s}=[\widetilde{D}-\bar{D}]+\varepsilon$, where $\bar{D}$ is the unconditional expectation of the actual dividend value, and $\varepsilon \sim N\left(0, \sigma_{\varepsilon}^{2}\right)$. The uninformed investors form rational expectations on $\widetilde{D}$ from the observation of $P$. In the market, there is also a measure 1 of correlated noise traders whose net demand of the risky asset is: $\widetilde{N} \sim N\left(0, \sigma_{N}^{2}\right)$. The initial allocation of the risky asset is:

$$
\mu a_{I}+(1-\mu) a_{U}+a_{N}=A
$$

where $a_{I}, a_{U}$ and $a_{N}$ denote the possessions of the informed investors, uninformed investors and noise traders, respectively, and $A$ denotes the fixed total amount of the shares. All traders have an identical endowment $a_{0}$ of the risk-free asset which they can trade against the risky asset at a unit price in the first period.

The informed investors form their expectation of $\widetilde{D}$ based on their private signal $\widetilde{s}$ as follows:

$$
E[\widetilde{D} \mid \widetilde{s}]=\bar{D}+\beta[\widetilde{s}]
$$

where $\beta$ is the informed investors' statistical prediction coefficient. According to the OLS method, the prediction coefficient is derived as: $\beta=\frac{\operatorname{cov}(\widetilde{D}, \widetilde{s})}{\operatorname{var}(\widetilde{s})}$. Noting that: $\operatorname{cov}(\widetilde{D}, \varepsilon)=0$, the prediction coefficient reads: 


$$
\beta=\frac{\sigma_{D}^{2}}{\sigma_{D}^{2}+\sigma_{\varepsilon}^{2}}
$$

where $\sigma_{D}^{2}$ is the variance of the dividend and $\sigma_{D}^{2}+\sigma_{\varepsilon}^{2}=\sigma_{s}^{2}$ is the variance of the noisy signal $\widetilde{s}$. Obviously, $0<\beta<1$, which means that the closer $\beta$ is to zero the vaguer are the predictions. The variance of the informed investors' prediction error is then calculated as $\sigma_{I}^{2}=\frac{\operatorname{var}(\widetilde{D}) \operatorname{var}(\varepsilon)}{\operatorname{var}(\widetilde{s})}$ so that:

$$
\sigma_{I}^{2}=\frac{\sigma_{D}^{2} \sigma_{\varepsilon}^{2}}{\sigma_{D}^{2}+\sigma_{\varepsilon}^{2}}
$$

Equation (4) describes the informed investors' prediction uncertainty upon given information and can be interpreted as the ex post risk of the risky asset per share.

The uninformed investors' expectation on the dividend value per share $\widetilde{D}$ is based on the observed market price $P$ like:

$$
E[\widetilde{D} \mid P]=\bar{D}+\gamma[P-\bar{P}]
$$

where $\gamma$ is the uninformed investors' prediction coefficient and $\bar{P}$ is the unconditional expectation of $P$, that is the average price of the risky asset. The formation of the market price depends on the informed investors' private signal $\widetilde{s}$ and the net actions of noise traders $\widetilde{N}$. Recalling that $\widetilde{s}=[\widetilde{D}-\bar{D}]+\varepsilon$, the expected value of both $\widetilde{s}$ and $\widetilde{N}$ is zero. A tentative assumption is that $P$ and the two random variables are correlated like:

$$
P=\bar{P}+b \widetilde{s}+c \widetilde{N}
$$

where $\bar{P}$ is the constant term and parameters $b$ and $c$ refer to the sensitivity of the market price to exogenous shocks in $\widetilde{s}$ and $\widetilde{N}$, respectively. Thus, the uninformed investors' expectation of $\widetilde{D}$ reads:

$$
E[\widetilde{D} \mid P]=\bar{D}+\gamma[b \widetilde{s}+c \widetilde{N}]
$$

Referring to Equations (5) and (6), the uninformed investors' prediction coefficient under given information comes from: $\gamma=\frac{b \operatorname{cov}(\widetilde{s}, \widetilde{D})}{\operatorname{var}(b \widetilde{s})+\operatorname{var}(c \widetilde{N})}$, which gives:

$$
\gamma=\frac{b \sigma_{D}^{2}}{b^{2}\left(\sigma_{D}^{2}+\sigma_{\varepsilon}^{2}\right)+c^{2} \sigma_{N}^{2}}
$$

The exact value of $\gamma$ depends on $b$ and $c$, which are so far unknown but derivable from the market equilibrium. The variance of the uninformed investors' statistical prediction error is calculated as: $\sigma_{U}^{2}=\frac{\operatorname{var}(\widetilde{D}) \operatorname{var}(b \varepsilon)+\operatorname{var}(\widetilde{D}) \operatorname{var}(c \widetilde{N})}{\operatorname{var}(b \widetilde{s})+\operatorname{var}(c \widetilde{N})}=\frac{\sigma_{D}^{2} b^{2} \sigma_{\varepsilon}^{2}+\sigma_{D}^{2} c^{2} \sigma_{N}^{2}}{b^{2}\left(\sigma_{D}^{2}+\sigma_{\varepsilon}^{2}\right)+c^{2} \sigma_{N}^{2}}$, which can be written as:

$$
\sigma_{U}^{2}=\theta \sigma_{D}^{2}
$$

where

$$
\theta=\frac{b^{2} \sigma_{\varepsilon}^{2}+c^{2} \sigma_{N}^{2}}{b^{2}\left(\sigma_{D}^{2}+\sigma_{\varepsilon}^{2}\right)+c^{2} \sigma_{N}^{2}}
$$

Equation (8) presents the uninformed investors' uncertainty of prediction under given information, which can be interpreted as the ex post risk per share. Please note that while $0<\theta<1$ clearly holds, the exact value of $\theta$ depends again on the sensitivity parameters $b$ and $c$. 


\section{The Financial Market}

In the financial market, rational investors optimize their holdings of risky and risk-free assets to maximize expected utility from consumption. An informed investor's CARA optimization problem reads:

$$
E\left[u\left(\widetilde{C}_{I} \mid \widetilde{s}\right)\right]=-e^{-x_{I} E[\widetilde{D} \mid \tilde{s}]-\left[a_{0}-\left(x_{I}-a_{I}\right) P\right]+\frac{x_{I}^{2}}{2} \sigma_{I}^{2}}
$$

where the informed investor's consumption in period 2 is $\widetilde{C}_{I}=x_{I} \widetilde{D}+\left[a_{0}-\left(x_{I}-a_{I}\right] P, x_{I}\right.$ is the net demand for the risky asset per share, $a_{0}$ is the endowment of the risk-free asset and $a_{I}$ is the initial holdings of the risky asset per share. $E[\widetilde{D} \mid \widetilde{s}]$ is the expected dividend per share, and $\frac{x_{I}^{2}}{2} \sigma_{I}^{2}$ is the risk element under the assumption of normally distributed noise in the signal $\widetilde{s}$. Taking the first-order condition with respect to demand $x_{I}$ produces:

$$
E[\widetilde{D} \mid \widetilde{s}]-P-x_{I} \sigma_{I}^{2}=0
$$

and solving for $x_{I}$ leads to:

$$
x_{I}=\frac{E[\widetilde{D} \mid \widetilde{s}]-P}{\sigma_{I}^{2}}
$$

Equation (11) says that the informed investor's demand of the risky asset equals the gain from investing in the risky asset divided by the variance of the informed investors' prediction error.

An uninformed investor's CARA optimization problem reads:

$$
E\left[u\left(\widetilde{C}_{U} \mid P\right)\right]=-e^{-x_{U} E[\widetilde{D} \mid P]-\left[a_{0}-\left(x_{U}-a_{U}\right) P\right]+\frac{x_{U}^{2}}{2} \sigma_{U}^{2}}
$$

where the uninformed investor's consumption in period 2 is: $\widetilde{C}_{U}=x_{U} \widetilde{D}+\left[a_{0}-\left(x_{U}-a_{U}\right)\right] P$, $x_{U}$ is the net demand for the risky asset per share, $a_{0}$ is the endowment of the risk-free asset and $a_{U}$ is the initial holdings of the risky asset per share. The first-order condition with respect to $x_{U}$ is:

$$
E[\widetilde{D} \mid P]-P-x_{U} \sigma_{U}^{2}=0
$$

and solving for $x_{U}$ gives:

$$
x_{U}=\frac{E[\widetilde{D} \mid P]-P}{\sigma_{U}^{2}}
$$

By Equation (13), the uninformed investor's demand of the risky asset equals the gain from investing in the risky asset divided by the variance of the uninformed investors' prediction error.

As the market supply of the risky asset is given in Equation (1), the market clearing condition for the risky asset per share is:

$$
\mu x_{I}+(1-\mu) x_{U}+\widetilde{N}=A
$$

which, recalling Equations (2), (5), (6), (11) and (13), turns to:

$$
\mu\left[\frac{\bar{D}+\beta \widetilde{s}-(\bar{P}+b \widetilde{s}+c \widetilde{N})}{\sigma_{I}^{2}}\right]+(1-\mu)\left[\frac{\bar{D}+\gamma(b \widetilde{s}+c \widetilde{N})-(\bar{P}+b \widetilde{s}+c \widetilde{N})}{\sigma_{U}^{2}}\right]+\widetilde{N}=A
$$

In a Walrasian equilibrium where demand equals supply, the sum of the constants on the left-hand side of Equation (15) must equal the constant supply $A$ of the risky asset on the right-hand side, and the sum of the coefficients of $\widetilde{s}$ and $\widetilde{N}$ must be equal to zero. Therefore, $\bar{P}, b$ and $c$ in Equation (5) can now be solved. To calculate $\bar{P}$, pick the constant 
terms from Equation (15), manipulate, and get: $\mu(\bar{D}-\bar{P}) \sigma_{U}^{2}+(1-\mu) \sigma_{I}^{2}(\bar{D}-\bar{P})=\sigma_{I}^{2} \sigma_{U}^{2} A$. Solving for $\bar{P}$ gives:

$$
\bar{P}=\bar{D}-\frac{\sigma_{I}^{2} \sigma_{U}^{2}}{\mu \sigma_{U}^{2}+(1-\mu) \sigma_{I}^{2}} A
$$

Equation (16) says that the average price $\bar{P}$ equals the unconditional expectation on the dividend minus discount, which consists of the risk term multiplied by $A$. Please note that $A>0$ means that $\bar{D}>\bar{P}$. Note also that a very big value of $A$ would make $\bar{P}$ negative, which is not reasonable. This is a tolerable artifact of the otherwise most useful CARA utility function (Mendel and Shleifer 2012). On the other hand, $A<0$ would make $\bar{D}<\bar{P}$. This is reasonable because the investors would then be short the asset on average, thus requiring a higher $P$ to compensate for the risk in borrowing shares to sell. Moreover, if information were symmetric, $\mu=1$ or 0 , the average discount would be like: $-\sigma_{I}^{2} A$ or $-\sigma_{U}^{2} A$, respectively.

To calculate the values of the sensitivity parameters $b$ and $c$, use Equation (15) to pick the coefficients of $\widetilde{s}$ and $\widetilde{N}$. After manipulation:

$$
\begin{aligned}
& b=\frac{\mu \beta \sigma_{U}^{2}}{\mu \sigma_{U}^{2}+(1-\mu)(1-\gamma) \sigma_{I}^{2}} \\
& c=\frac{\sigma_{I}^{2} \sigma_{U}^{2}}{\mu \sigma_{U}^{2}+(1-\mu)(1-\gamma) \sigma_{I}^{2}}
\end{aligned}
$$

The signs of the sensitivity parameters $b$ and $c$ need closer scrutiny. By Equation (8), $\sigma_{U}^{2}$ depends on $\theta$, which in turn depends on $b$ and $c$ by Equation (9). To investigate the interdependency, divide $b$ in Equation (17) by $c$ in Equation (18) to get $\frac{b}{c}=\frac{\mu \beta}{\sigma_{I}^{2}}$ which, after using Equations (3) and (4), reduces to:

$$
\frac{b}{c}=\frac{\mu}{\sigma_{\varepsilon}^{2}}
$$

Taking squares on both sides yields: $b^{2}=\frac{c^{2} \mu^{2}}{\sigma_{\varepsilon}^{4}}$, and substitution into Equation (9) gives: $\theta=\frac{\mu^{2} \sigma_{\varepsilon}^{2} / \sigma_{\varepsilon}^{4}+\sigma_{N}^{2}}{\mu^{2}\left(\left(\sigma_{D}^{2}+\sigma_{\varepsilon}^{2}\right) / \sigma_{\varepsilon}^{4}\right)+\sigma_{N}^{2}}$. Multiply by $\frac{\sigma_{\varepsilon}^{4}}{\sigma_{\varepsilon}^{4}}$ and manipulate to write:

$$
\theta=\frac{\mu^{2} \sigma_{\varepsilon}^{2}+\sigma_{\varepsilon}^{4} \sigma_{N}^{2}}{\mu^{2}\left(\sigma_{D}^{2}+\sigma_{\varepsilon}^{2}\right)+\sigma_{\varepsilon}^{4} \sigma_{N}^{2}}
$$

Equation (20) confirms that $0<\theta<1$. As it is given by underlying parameters, $\sigma_{U}^{2}$ in Equation (8) is also given by them. Please note that without the noise trader effect: $\theta=\frac{\sigma_{\varepsilon}^{2}}{\sigma_{D}^{2}+\sigma_{\varepsilon}^{2}}$. Then, recalling Equations (4) and (8), $\theta \sigma_{D}^{2}=\sigma_{I}^{2}=\sigma_{U}^{2}$. Hence, Equation (20) demonstrates that the difference between the informed and uninformed investors' variance of prediction errors is due to noise trading.

Moreover, Equations (17) and (18) show that the signs of $b$ and $c$ depend on $\gamma$, and Equation (7): $\gamma=\frac{b \sigma_{D}^{2}}{b^{2}\left(\sigma_{D}^{2}+\sigma_{\varepsilon}^{2}\right)+c^{2} \sigma_{N}^{2}}$ says that $\gamma$ depends on $b$ and $c$. Write Equation (7) as $\gamma b^{2}\left(\sigma_{D}^{2}+\sigma_{\varepsilon}^{2}\right)+\gamma c^{2} \sigma_{N}^{2}=b \sigma_{D}^{2}$, and use Equations (17) and (18) to substitute for $b$ and $c$. This yields, after some manipulation:

$$
\frac{\gamma \mu^{2} \beta^{2} \sigma_{U}^{4}\left(\sigma_{D}^{2}+\sigma_{\varepsilon}^{2}\right)+\gamma \sigma_{I}^{4} \sigma_{U}^{4} \sigma_{N}^{2}}{\mu \sigma_{U}^{2}+(1-\mu)(1-\gamma) \sigma_{I}^{2}}=\frac{\mu \beta \sigma_{U}^{2} \sigma_{D}^{2}}{\mu \sigma_{U}^{2}+(1-\mu)(1-\gamma) \sigma_{I}^{2}}
$$

Multiply through by $\mu \sigma_{U}^{2}+(1-\mu)(1-\gamma) \sigma_{I}^{2}$, use: $\beta\left(\sigma_{D}^{2}+\sigma_{\varepsilon}^{2}\right)=\sigma_{D}^{2}$ from Equation (3) and manipulate to obtain $\gamma\left[\mu^{2} \beta \sigma_{D}^{2} \sigma_{U}^{4}+\mu \beta \sigma_{U}^{2} \sigma_{D}^{2}(1-\mu) \sigma_{I}^{2}+\sigma_{I}^{4} \sigma_{U}^{4} \sigma_{N}^{2}\right]=\beta \mu \sigma_{U}^{2} \sigma_{D}^{2}$ 
$\left[\mu \sigma_{U}^{2}+(1-\mu) \sigma_{I}^{2}\right]$. Solve for $\gamma$ to get $\gamma=\frac{\mu \beta\left[\mu \sigma_{U}^{2}+(1-\mu) \sigma_{I}^{2}\right]}{\mu \beta\left[\mu \sigma_{U}^{2}+(1-\mu) \sigma_{I}^{2}\right]+\left(\sigma_{I}^{4} \sigma_{U}^{2} \sigma_{N}^{2}\right) / \sigma_{D}^{2}}$. Finally, use $\sigma_{D}^{2}=$ $\sigma_{U}^{2} / \theta$ from Equation (8) and write:

$$
\gamma=\frac{\mu \beta\left[\mu \sigma_{U}^{2}+(1-\mu) \sigma_{I}^{2}\right]}{\mu \beta\left[\mu \sigma_{U}^{2}+(1-\mu) \sigma_{I}^{2}\right]+\theta \sigma_{I}^{4} \sigma_{N}^{2}}
$$

Equation (21) confirms that $0<\gamma<1$ (note that $\sigma_{N}^{2} \rightarrow 0$ makes $\gamma \rightarrow 1$ ). Therefore, $b$ and $c$ in Equations (17) and (18) are clearly positive. Plug Equation (21) into (17), manipulate and get:

$$
b=\frac{\beta \mu \sigma_{U}^{2}\left\{\mu \beta\left[\mu \sigma_{U}^{2}+(1-\mu) \sigma_{I}^{2}\right]+\theta \sigma_{I}^{4} \sigma_{N}^{2}\right\}}{\mu \sigma_{U}^{2}\left\{\mu \beta\left[\mu \sigma_{U}^{2}+(1-\mu) \sigma_{I}^{2}\right]+\theta \sigma_{I}^{4} \sigma_{N}^{2}\right\}+(1-\mu) \sigma_{I}^{2} \theta \sigma_{I}^{4} \sigma_{N}^{2}}
$$

Likewise, plug Equation (21) into (18) and get:

$$
c=\frac{\sigma_{I}^{2} \sigma_{U}^{2}\left\{\mu \beta\left[\mu \sigma_{U}^{2}+(1-\mu) \sigma_{I}^{2}\right]+\theta \sigma_{I}^{4} \sigma_{N}^{2}\right\}}{\mu \sigma_{U}^{2}\left\{\mu \beta\left[\mu \sigma_{U}^{2}+(1-\mu) \sigma_{I}^{2}\right]+\theta \sigma_{I}^{4} \sigma_{N}^{2}\right\}+(1-\mu) \sigma_{I}^{2} \theta \sigma_{I}^{4} \sigma_{N}^{2}}
$$

Finally, substitute Equations (16), (22) and (23) into Equation (5), which leads to:

$$
\begin{aligned}
P=\widetilde{D}- & \frac{\sigma_{I}^{2} \sigma_{U}^{2}}{\mu \sigma_{U}^{2}+(1-\mu) \sigma_{I}^{2}} A+\frac{\mu \beta \sigma_{U}^{2}\left\{\mu \beta\left[\mu \sigma_{U}^{2}+(1-\mu) \sigma_{I}^{2}\right]+\theta \sigma_{I}^{4} \sigma_{N}^{2}\right\}}{\mu \sigma_{U}^{2}\left\{\mu \beta\left[\mu \sigma_{U}^{2}+(1-\mu) \sigma_{I}^{2}\right]+\theta \sigma_{I}^{4} \sigma_{N}^{2}\right\}+(1-\mu) \sigma_{I}^{2} \theta \sigma_{I}^{4} \sigma_{N}^{2}} \widetilde{\mathcal{S}} \\
& +\frac{\sigma_{I}^{2} \sigma_{U}^{2}\left\{\mu \beta\left[\mu \sigma_{U}^{2}+(1-\mu) \sigma_{I}^{2}\right]+\theta \sigma_{I}^{4} \sigma_{N}^{2}\right\}}{\mu \sigma_{U}^{2}\left\{\mu \beta\left[\mu \sigma_{U}^{2}+(1-\mu) \sigma_{I}^{2}\right]+\theta \sigma_{I}^{4} \sigma_{N}^{2}\right\}+(1-\mu) \sigma_{I}^{2} \theta \sigma_{I}^{4} \sigma_{N}^{2}} \widetilde{N}
\end{aligned}
$$

Equation (24) presents the formation of the market price of the risky asset. Since the sensitivity parameters $b$ and $c$ are unambiguously positive, the rational investors' market reactions follow the signs of the shocks in $\widetilde{s}$ and $\widetilde{N}$ and the market price responds accordingly.

\section{The Central Bank}

To incorporate the central bank into the model, suppose that it launches the leaning against the bubble policy in the financial market. We use the acronym LAB for the policy, which reacts to asset price movements by buying the risky asset (in exchange of the riskless asset) when its price is too low and selling it when the price is too high.

The policy rule is attached to the deviations of the market price from the average price of the risky asset. If the market price is higher than the average price, the rule implies a reduction of the bank's holdings of the risky asset so that the increased market supply induces the market price to fall, and vice versa. The central bank's policy function reads:

$$
x_{Q}=q_{0}-q_{1}(P-\bar{P})
$$

where $x_{Q}$ is the central bank's net demand per share of the risky asset in the first period, $q_{0}$ is its net holdings of the risky asset when the market price $P$ equals its average value $\bar{P}$, and $q_{1}>0$ is a sensitivity parameter that tells how much the central bank sells or buys in the asset market if $P$ deviates one unit per share from $\bar{P}$. Please note that $q_{0}$ and $q_{1}$ are independent of each other. Upon introduction, rational investors take the policy rule it into account in their market decisions (Sargent and Wallace 1975). The market clearing condition becomes:

$$
\mu x_{I}+(1-\mu) x_{U}+\widetilde{N}+x_{Q}=A
$$

Recalling Equations (5), (15) and (25), the market clears when:

$$
\begin{aligned}
\mu\left[\frac{\bar{D}+\beta \widetilde{s}-(\bar{P}+b \widetilde{s}+c \widetilde{N})}{\sigma_{I}^{2}}\right]+ & (1-\mu)\left[\frac{\bar{D}+\gamma(b \widetilde{s}+c \widetilde{N})-(\bar{P}+b \widetilde{s}+c \widetilde{N})}{\sigma_{U}^{2}}\right]+\widetilde{N}+q_{0} \\
& -q_{1}(b \widetilde{s}+c \widetilde{N})=A
\end{aligned}
$$


The constant $\bar{P}$ and the sensitivity parameters $b$ and $c$ under the LAW policy can now be solved by repeating the same procedure as in the policy-free case above. This produces:

$$
\begin{gathered}
\bar{P}=\bar{D}+\frac{\sigma_{I}^{2} \sigma_{U}^{2}}{\mu \sigma_{U}^{2}+(1-\mu) \sigma_{I}^{2}}\left(q_{0}-A\right) \\
\hat{b}=\frac{\mu \beta \sigma_{U}^{2}}{\mu \sigma_{U}^{2}+(1-\mu)(1-\hat{\gamma}) \sigma_{I}^{2}+q_{1} \sigma_{I}^{2} \sigma_{U}^{2}} \\
\hat{c}=\frac{\sigma_{I}^{2} \sigma_{U}^{2}}{\mu \sigma_{U}^{2}+(1-\mu)(1-\hat{\gamma}) \sigma_{I}^{2}+q_{1} \sigma_{I}^{2} \sigma_{U}^{2}}
\end{gathered}
$$

The uninformed investors' prediction coefficient is now denoted by $\hat{\gamma}$. It can be calculated by substituting $\hat{b}$ and $\hat{c}$ from Equations (29) and (30) into Equation (7): $\gamma=$ $\frac{b \sigma_{D}^{2}}{b^{2}\left(\sigma_{D}^{2}+\sigma_{\varepsilon}^{2}\right)+c^{2} \sigma_{N}^{2}}$, which yields, after some manipulation, $\hat{\gamma}\left[\mu^{2} \beta^{2} \sigma_{U}^{2}\left(\sigma_{D}^{2}+\sigma_{\varepsilon}^{2}\right)+\sigma_{I}^{4} \sigma_{U}^{2} \sigma_{N}^{2}+\right]$ $\left[\mu \beta \sigma_{D}^{2}(1-\mu) \sigma_{I}^{2}\right]=\mu \beta \sigma_{D}^{2}\left[\mu \sigma_{U}^{2}+(1-\mu) \sigma_{I}^{2}+q_{1} \sigma_{I}^{2} \sigma_{U}^{2}\right]$. Solving for $\hat{\gamma}$, and using Equation (8) gives $\hat{\gamma}=\frac{\mu \beta\left[\mu \sigma_{U}^{2}+(1-\mu) \sigma_{I}^{2}+q_{1} \sigma_{I}^{2} \sigma_{U}^{2}\right]}{\mu \beta\left[\mu \theta \beta\left(\sigma_{D}^{2}+\sigma_{\varepsilon}^{2}\right)+(1-\mu) \sigma_{I}^{2}\right]+\theta \sigma_{I}^{4} \sigma_{N}^{2}}$. Use Equation (3) in the first term in the denominator to substitute for $\beta\left(\sigma_{D}^{2}+\sigma_{\varepsilon}^{2}\right)=\sigma_{D}^{2}$ and then use Equation (8) to substitute for $\theta \sigma_{D}^{2}=\sigma_{U}^{2}$. This produces:

$$
\hat{\gamma}=\frac{\mu \beta\left[\mu \sigma_{U}^{2}+(1-\mu) \sigma_{I}^{2}+q_{1} \sigma_{I}^{2} \sigma_{U}^{2}\right]}{\mu \beta\left[\mu \sigma_{U}^{2}+(1-\mu) \sigma_{I}^{2}\right]+\theta \sigma_{I}^{4} \sigma_{N}^{2}}
$$

which is clearly positive. Plug Equation (31) into Equation (29) and get, after manipulation:

$$
\hat{b}=\frac{\mu \beta \sigma_{U}^{2}\left\{\mu \beta\left[\mu \sigma_{U}^{2}+(1-\mu) \sigma_{I}^{2}\right]+\theta \sigma_{I}^{4} \sigma_{N}^{2}\right\}}{\mu \sigma_{U}^{2}\left\{\mu \beta\left[\mu \sigma_{U}^{2}+(1-\mu) \sigma_{I}^{2}\right]+\theta \sigma_{I}^{4} \sigma_{N}^{2}\right\}+(1-\mu) \sigma_{I}^{2} \theta \sigma_{I}^{4} \sigma_{N}^{2}+q_{1} \sigma_{I}^{2} \sigma_{U}^{2}\left(\mu^{2} \beta \sigma_{U}^{2}+\theta \sigma_{I}^{4} \sigma_{N}^{2}\right)}
$$

which is positive. Likewise, plug Equation (30) into Equation (29), manipulate and obtain:

$$
\hat{c}=\frac{\sigma_{I}^{2} \sigma_{U}^{2}\left\{\mu \beta\left[\mu \sigma_{U}^{2}+(1-\mu) \sigma_{I}^{2}\right]+\theta \sigma_{I}^{4} \sigma_{N}^{2}\right\}}{\mu \sigma_{U}^{2}\left\{\mu \beta\left[\mu \sigma_{U}^{2}+(1-\mu) \sigma_{I}^{2}\right]+\theta \sigma_{I}^{4} \sigma_{N}^{2}\right\}+(1-\mu) \sigma_{I}^{2} \theta \sigma_{I}^{4} \sigma_{N}^{2}+q_{1} \sigma_{I}^{2} \sigma_{U}^{2}\left(\mu^{2} \beta \sigma_{U}^{2}+\theta \sigma_{I}^{4} \sigma_{N}^{2}\right)}
$$

which is also positive. Substituting Equations (32) and (33) into Equation (5), the market price formula under the LAB policy reads:

$$
\begin{aligned}
& P=\widetilde{D}+\frac{\sigma_{I}^{2} \sigma_{U}^{2}}{\mu \sigma_{U}^{2}+(1-\mu) \sigma_{I}^{2}}\left(q_{0}-A\right) \\
& +\frac{\mu \beta \sigma_{U}^{2}\left\{\mu \beta\left[\mu \sigma_{U}^{2}+(1-\mu) \sigma_{I}^{2}\right]+\theta \sigma_{I}^{4} \sigma_{N}^{2}\right\}}{\mu \sigma_{U}^{2}\left\{\mu \beta\left[\mu \sigma_{U}^{2}+(1-\mu) \sigma_{I}^{2}\right]+\theta \sigma_{I}^{4} \sigma_{N}^{2}\right\}+(1-\mu) \sigma_{I}^{2} \theta \sigma_{I}^{4} \sigma_{N}^{2}+q_{1} \sigma_{I}^{2} \sigma_{U}^{2}\left(\mu^{2} \beta \sigma_{U}^{2}+\theta \sigma_{I}^{4} \sigma_{N}^{2}\right)} \widetilde{s} \\
& +\frac{\sigma_{I}^{2} \sigma_{U}^{2}\left\{\mu \beta\left[\mu \sigma_{U}^{2}+(1-\mu) \sigma_{I}^{2}\right]+\theta \sigma_{I}^{4} \sigma_{N}^{2}\right\}}{\mu \sigma_{U}^{2}\left\{\mu \beta\left[\mu \sigma_{U}^{2}+(1-\mu) \sigma_{I}^{2}\right]+\theta \sigma_{I}^{4} \sigma_{N}^{2}\right\}+(1-\mu) \sigma_{I}^{2} \theta \sigma_{I}^{4} \sigma_{N}^{2}+q_{1} \sigma_{I}^{2} \sigma_{U}^{2}\left(\mu^{2} \beta \sigma_{U}^{2}+\theta \sigma_{I}^{4} \sigma_{N}^{2}\right)} \widetilde{N}
\end{aligned}
$$

\section{Results}

Proposition 1. The introduction of the LAB policy makes the risky asset's market price per share less sensitive to information shocks.

Proof. To examine the effects of the central bank's intervention on the price sensitivity parameters $b$ and $c$, compare them without and with the policy. The impact of the intervention on $b$ can be seen by comparing Equations (22) and (32). Postulating that $b>\hat{b}$ reads:

$$
\frac{\beta \mu \sigma_{U}^{2}\left\{\mu \beta\left[\mu \sigma_{U}^{2}+(1-\mu) \sigma_{I}^{2}\right]+\theta \sigma_{I}^{4} \sigma_{N}^{2}\right\}}{\mu \sigma_{U}^{2}\left\{\mu \beta\left[\mu \sigma_{U}^{2}+(1-\mu) \sigma_{I}^{2}\right]+\theta \sigma_{I}^{4} \sigma_{N}^{2}\right\}+(1-\mu) \sigma_{I}^{2} \theta \sigma_{I}^{4} \sigma_{N}^{2}}>\frac{\mu \beta \sigma_{U}^{2}\left\{\mu \beta\left[\mu \sigma_{U}^{2}+(1-\mu) \sigma_{I}^{2}\right]+\theta \sigma_{I}^{4} \sigma_{N}^{2}\right\}}{\mu \sigma_{U}^{2}\left\{\mu \beta\left[\mu \sigma_{U}^{2}+(1-\mu) \sigma_{I}^{2}\right]+\theta \sigma_{I}^{4} \sigma_{N}^{2}\right\}+(1-\mu) \sigma_{I}^{2} \theta \sigma_{I}^{4} \sigma_{N}^{2}+q_{1} \sigma_{I}^{2} \sigma_{U}^{2}\left(\mu^{2} \beta \sigma_{U}^{2}+\theta \sigma_{I}^{4} \sigma_{N}^{2}\right)}
$$


After straightforward manipulation, it reduces to:

$$
q_{1} \sigma_{I}^{2} \sigma_{U}^{2}\left(\mu^{2} \beta \sigma_{U}^{2}+\theta \sigma_{I}^{4} \sigma_{N}^{2}\right)>0
$$

The positive sign is definitive since $0<\theta<1$ is verified by Equation (20) in terms of underlying parameters. Thus, the expression confirms that $b>\hat{b}$. Postulating that $c>\hat{c}$, which reduces again to:

$$
q_{1} \sigma_{I}^{2} \sigma_{U}^{2}\left(\mu^{2} \beta \sigma_{U}^{2}+\theta \sigma_{I}^{4} \sigma_{N}^{2}\right)>0
$$

Therefore, $c>\hat{c}$ is also confirmed.

The conclusion is that the implementation of the central banks' LAB policy makes market price formation less sensitive to private signals $(\hat{b}<b)$ and noise traders' net transactions $(\hat{c}<c)$. Q.E.D.

Proposition 2. The introduction of the LAB policy makes the risky asset's market price and the average price converge, thus reducing the variance of the market price.

Proof. By Equation (5), the market price of the risky asset reads: $P=\bar{P}+b \widetilde{s}+c \widetilde{N}$ so that the deviation of the market price from the average price under the policy is:

$$
P-\bar{P}=\hat{b} \widetilde{s}+\hat{c} \widetilde{N}
$$

Since the proof of Proposition 1 tells that: $\hat{b}<b$ and $\hat{c}<c$, the difference $P-\bar{P}$ must get smaller under the policy. Moreover, the variance of the market price $P$ is calculated as $\operatorname{var}(P)=\operatorname{var}(b \widetilde{s})+\operatorname{var}(c \widetilde{N})$, which produces:

$$
\sigma_{P}^{2}=b^{2}\left(\sigma_{D}^{2}+\sigma_{\varepsilon}^{2}\right)+c^{2} \sigma_{N}^{2}
$$

Under the policy the same reads:

$$
\sigma_{P}^{2}=\hat{b}^{2}\left(\sigma_{D}^{2}+\sigma_{\varepsilon}^{2}\right)+\hat{c}^{2} \sigma_{N}^{2}
$$

Since $\hat{b}<b$ and $\hat{c}<c$ hold by the proof of Proposition 1 , the introduction of the policy clearly reduces the price variance of the risky asset.

The conclusion is that the implementation of the central banks' LAB policy drives the market price of the risky asset closer to its average thus reducing the variance of the market price. Q.E.D.

Proposition 3. The introduction of the LAB policy does not affect the informed investors' prediction coefficient, but augments that of the uninformed investors.

Proof. The informed investors' prediction coefficient is given by Equation (3):

$$
\beta=\frac{\sigma_{D}^{2}}{\sigma_{D}^{2}+\sigma_{\varepsilon}^{2}}
$$

which defines the coefficient purely in terms of the underlying parameters. Therefore, the policy has no effects on the informed investors' prediction coefficient.

To examine the impact of the policy on the uninformed investors' prediction coefficient, compare $\gamma$ from Equation (21) $\frac{\mu \beta\left[\mu \sigma_{U}^{2}+(1-\mu) \sigma_{I}^{2}\right]}{\mu \beta\left[\mu \sigma_{U}^{2}+(1-\mu) \sigma_{I}^{2}\right]+\theta \sigma_{I}^{4} \sigma_{N}^{2}}$ to $\hat{\gamma}$ in Equation (29) $\frac{\mu \beta\left[\mu \sigma_{U}^{2}+(1-\mu) \sigma_{I}^{2}+q_{1} \sigma_{I}^{2} \sigma_{U}^{2}\right]}{\mu \beta\left[\mu \sigma_{U}^{2}+(1-\mu) \sigma_{I}^{2}\right]+\theta \sigma_{I}^{4} \sigma_{N}^{2}}$. Postulate that $\hat{\gamma}>\gamma$, calculate the difference, and get:

$$
\hat{\gamma}-\gamma=\frac{\mu \beta q_{1} \sigma_{I}^{2} \sigma_{U}^{2}}{\mu \beta\left[\mu \sigma_{U}^{2}+(1-\mu) \sigma_{I}^{2}\right]+\theta \sigma_{I}^{4} \sigma_{N}^{2}}>0
$$


Since $\hat{\gamma}>\gamma$ clearly holds, the introduction of the policy makes the uninformed investors' prediction coefficient grow.

In summary, the central bank's LAB policy does not affect the informed investors' prediction coefficient but magnifies that of the uninformed investors $(\hat{\gamma}>\gamma)$. Q.E.D.

Proposition 4. The fall in the sensitivity parameters is cancelled out by the rise in the uninformed investors' prediction coefficient to keep the expected value of the risky asset's dividend unaltered.

Proof. Recalling Equation (6), the uninformed investors' expectation of the value of the risky asset's dividend reads: $E[\widetilde{D} \mid P]=\bar{D}+\gamma[b \widetilde{\mathcal{S}}+c \widetilde{N}]$. Under the LAB policy, recalling Equations (32) and (33), it turns to:

$$
E[\widetilde{D} \mid P]=\bar{D}+\gamma\left[\mu \beta \sigma_{U}^{2} \widetilde{s}+\sigma_{I}^{2} \sigma_{U}^{2} \widetilde{N}\right] B
$$

where $B=\frac{\mu \beta\left[\mu \sigma_{U}^{2}+(1-\mu) \sigma_{I}^{2}\right]+\theta \sigma_{I}^{4} \sigma_{N}^{2}}{\mu \sigma_{U}^{2}\left\{\mu \beta\left[\mu \sigma_{U}^{2}+(1-\mu) \sigma_{I}^{2}\right]+\theta \sigma_{I}^{4} \sigma_{N}^{2}\right\}+(1-\mu) \sigma_{I}^{2} \theta \sigma_{I}^{4} \sigma_{N}^{2}+q_{1} \sigma_{I}^{2} \sigma_{U}^{2}\left(\mu^{2} \beta \sigma_{U}^{2}+\theta \sigma_{I}^{4} \sigma_{N}^{2}\right)}$ denotes the coefficient which is under the influence of the $\mathrm{LAB}$ policy and: $\mu \beta \sigma_{U}^{2} B=\hat{b}, \sigma_{I}^{2} \sigma_{U}^{2} B=\hat{c}$. Since the proof of Proposition 1 says the LAB policy makes the sensitivity parameters diminish, the constancy of the uninformed investors' expectations on $\widetilde{D}$ necessitates that the growth of $\gamma$, recorded in the proof of Proposition 3, balances the effect. This implies that $\gamma \hat{b}$ and $\gamma \hat{c}$ should be constant and thus independent of policy. Divide $\hat{b}=\mu \beta \sigma_{U}^{2} B$ by $\hat{c}=\sigma_{I}^{2} \sigma_{U}^{2} B$ and manipulate to find that $\frac{\hat{b}}{\hat{c}}=\frac{\mu}{\sigma_{\varepsilon}^{2}}$. Therefore, Equation (19) holds also under the LAB policy and it suffices to apply Equation (7), $\gamma=\frac{b \sigma_{D}^{2}}{b^{2}\left(\sigma_{D}^{2}+\sigma_{\varepsilon}^{2}\right)+c^{2} \sigma_{N}^{2}}$, to write:

$$
\hat{b} \gamma=\frac{\hat{b} \sigma_{D}^{2}}{\hat{b}^{2}\left(\sigma_{D}^{2}+\sigma_{\varepsilon}^{2}\right)+\hat{c}^{2} \sigma_{N}^{2}}
$$

Using $\hat{b}=\mu$ and $\hat{c}=\sigma_{\varepsilon}^{2}$ produces:

$$
\hat{b} \gamma=\frac{\mu^{2} \sigma_{D}^{2}}{\mu^{2}\left(\sigma_{D}^{2}+\sigma_{\varepsilon}^{2}\right)+\sigma_{\varepsilon}^{4} \sigma_{N}^{2}}
$$

which means that $\gamma \hat{b}$ is constant and independent of policy. Likewise, using $\hat{b}=\mu$ and $\hat{c}=$ $\sigma_{\varepsilon}^{2}\left(\right.$ and $\left.\hat{c} \hat{b}=\mu \sigma_{\varepsilon}^{2}\right)$ produces:

$$
\hat{c} \gamma=\frac{\mu \sigma_{\varepsilon}^{2} \sigma_{D}^{2}}{\mu^{2}\left(\sigma_{D}^{2}+\sigma_{\varepsilon}^{2}\right)+\sigma_{\varepsilon}^{4} \sigma_{N}^{2}}
$$

meaning that $\gamma \hat{c}$ is also constant and independent of policy.

The conclusion is that not only the uninformed but also the informed investors' expectations of the risky asset's dividend value remain unaltered under the LAB policy because the rise in the uninformed investors' prediction coefficient perfectly cancels out the fall in the sensitivity parameters. Q.E.D.

Proposition 5. The introduction of the central bank's $L A B$ wind does not affect rational investors' risk per share.

Proof. The variance of prediction error measures ex post risk, that is, risk remaining after the agents have received their information. The informed investors' variance of prediction error is given by Equation (4):

$$
\sigma_{I}^{2}=\frac{\sigma_{D}^{2} \sigma_{\varepsilon}^{2}}{\sigma_{D}^{2}+\sigma_{\varepsilon}^{2}}
$$


Since $\sigma_{I}^{2}$ is determined purely by underlying parameters, the policy does not affect the informed investors' ex-post risk. The uninformed investors' variance of prediction error is given by Equation (8), $\sigma_{U}^{2}=\theta \sigma_{D}^{2}$, and use of Equation (20) yields:

$$
\sigma_{U}^{2}=\frac{\mu^{2} \sigma_{\varepsilon}^{2}+\sigma_{\varepsilon}^{4} \sigma_{N}^{2}}{\mu^{2}\left(\sigma_{D}^{2}+\sigma_{\varepsilon}^{2}\right)+\sigma_{\varepsilon}^{4} \sigma_{N}^{2}} \sigma_{D}^{2}
$$

Since $\sigma_{U}^{2}$ depends only on underlying parameters, the uninformed investors' variance of prediction error is independent of the policy.

The conclusion is that neither the informed and uninformed investors' variance of prediction error is affected by the LAB policy. Since the risk aversion coefficient equals one under the CARA assumption, it can be interpreted that the LAB policy has no effects on rational investors' ex post risk per share.Q.E.D.

\section{Conclusions and Discussion}

This paper was a theoretical analysis of a Walrasian financial market composed of informed and uninformed constant absolute risk-averse (CARA) rational investors, and noise traders. The rational investors were assumed to trade their initial holdings of risk-free assets for risky assets in the first period to maximize their consumption utility in the second period. The properties of the model were first analyzed in a policy-free context, and then compared to the situation where the central bank implements a direct leaning against the bubble (LAB) policy to systematically control possible overpricing or underpricing of the risky asset.

The analyses provided many insightful observations. First, it was found that the implementation of the LAB policy makes market price formation less sensitive to private fundament-based signals and noise traders' sentiment-based moves. Second, the policy was found to drive the risky asset's market price closer to its average thus reducing price variance. Third, it was shown that the policy does not affect the informed investors' prediction coefficient but magnifies that of the uninformed investors. Fourth, it was clarified that the rise in the uninformed investors' prediction coefficient cancels out the fall in the sensitivity parameters thus keeping both informed and uninformed investors' expectations on the risky asset's dividend unaltered. The final observation was that rational investors' variance of prediction error is not affected by the policy, which implies that rational investors' ex post risk per share is independent of the policy.

A general conclusion from the theoretical investigations is that the central bank's leaning against the bubble policy as we describe it is an effective automatic price stabilizer in the financial market. The effects of the policy channel through rational investors' market behavior, which leads to the reduction of price volatility in the financial market.

Some practical limitations on the application of our LAB policy must be addressed, too. First, the Walrasian framework assumes perfect markets, which sounds contradictory to the assumption of asymmetric information. Despite the minor inconvenience, the model is widely used in that context, but the more profound question still remains: is the price mechanism really efficient in the financial market? In our model, only the central bank can manipulate asset prices, but real life shows that there are also other powerful agents in the market. Second, the LAB policy should be able to control both credit and exuberance driven bubbles, but what if a sudden price movement is due to a positive productivity shock?

In any case, our model opens paths for further investigations. For example, it would be insightful to investigate the policy effects on ex ante risk, that is the variance of the difference between the random dividend and the market price. Another interesting question concerns the uninformed investors' demand for the risky asset, particularly the slope of their demand curve. Moreover, overpricing or underpricing could be reasoned, noise traders could be modeled better, various bubble elements familiar from the behavioral finance literature could be included and so on. Empirical analyses are naturally needed to shed light on the practical efficacy of the policy. 
Author Contributions: Conceptualization, H.L. and J.I.; methodology, H.L. and J.I.; software, C.L.C.; validation, H.L., J.I. and C.-L.C.; formal analysis, H.L., J.I. and C.-L.C.; investigation, H.L. and J.I.; resources, H.L., J.I. and C.-L.C.; data curation, H.L., J.I. and C.-L.C.; writing-original draft preparation, H.L. and J.I.; writing-review and editing, H.L., J.I. and C.-L.C.; visualization, H.L., J.I. and C.-L.C.; supervision, H.L., J.I. and C.-L.C.; project administration, H.L., J.I. and C.-L.C.; funding acquisition, C.-L.C. All authors have read and agreed to the published version of the manuscript.

Funding: Financial support from Ministry of Science and Technology (MOST), Taiwan, is acknowledged. The authors are most grateful to Michael McAleer for his helpful comments.

Conflicts of Interest: The authors declare no conflict of interest.

\section{References}

Admati, Anat. 1985. A noisy rational expectations equilibrium for multi-asset securities markets. Econometrica 53: 629-57. [CrossRef]

Allen, Franklin, Stephen Morris, and Hyun Shin. 2006. Beauty contests and iterated expectations in asset markets. Review of Financial Studies 19: 719-52. [CrossRef]

Bacchetta, Philippe, and Eric Van Wincoop. 2008. Higher order expectations in asset pricing. Journal of Money, Credit and Banking 40: 837-65. [CrossRef]

Bean, Charles. 2004. Asset prices, financial stability, and monetary policy. American Economic Review 94: 14-18. [CrossRef]

Bernanke, Ben, and Mark Gertler. 2001. Should central banks respond to movements in asset prices? American Economic Review 91: 253-57. [CrossRef]

Brunnermeier, Markus, and Isabel Schnabel. 2016. Bubbles and central banks. In Central Banks at a Crossroads. Cambridge: Cambridge University Press, pp. 493-562.

Campbell, John, and Albert Kyle. 1993. Smart money, noise trading and stock price behavior. Review of Economic Studies 60: 1-34. [CrossRef]

Ciccarone, Giuseppe, Giuli Francesco, Enrico Marchetti, and Massimiliano Tancioni. 2020. Leaning against the bubble. Can theoretical models match the empirical evidence? In MPRA Paper. No. 105004. Munich: University Library LMU Munich.

Conlon, John. 2015. Should central banks burst bubbles? Some microeconomic issues. Economic Journal 125: 141-61. [CrossRef]

De Bondt, Werner, and Richard Thaler. 1985. Does the stock market overreact? Journal of Finance 40: 793-805. [CrossRef]

Dong, Fen, Jianjun Miao, and Pengfei Wang. 2020. Asset bubbles and monetary policy. Review of Economic Dynamics 37: S68-S98. [CrossRef]

Gali, Jordi. 2014. Monetary policy and rational asset price bubbles. American Economic Review 104: 721-52. [CrossRef]

Greenspan, Alan. 2002. Economic Volatility, Speech at a Symposium Sponsored by the Federal Reserve Bank of Kansas City. Jackson Hole. Washington, DC: The Federal Reserve Board.

Greenspan, Alan. 2004. Risk and uncertainty in monetary policy. American Economic Review 94: 33-40. [CrossRef]

Grossman, Sanford, and Joseph Stiglitz. 1980. On the impossibility of informationally efficient markets. American Economic Review 70: 393-408.

Hellwig, Martin. 1980. On the aggregation of information in competitive markets. Journal of Economic Theory 22: 477-98. [CrossRef]

Hirshleifer, David. 2015. Behavioral Finance. Annual Review of Financial Economics 7: 133-59. [CrossRef]

Ilomäki, Jukka, and Hannu Laurila. 2018. The noise trader effect in a Walrasian market. Advances in Decision Sciences 22: 1-14.

Ilomäki, Jukka, and Hannu Laurila. 2020. Inflation and risky investments. Journal of Risk and Financial Management 13: 329.

Ilomäki, Jukka, and Hannu Laurila. 2021. Leaning against the wind policy and animal spirits in a general equilibrium model. International Journal of Finance $\mathcal{E}$ Economics 26: 2325-34.

Mendel, Brock, and Andrei Shleifer. 2012. Chasing noise. Journal of Financial Economics 104: 203-320. [CrossRef]

Mishkin, Frederic. 2017. Rethinking monetary policy after the crisis. Journal of International Money and Finance 73: 252-74. [CrossRef]

Posen, Adam. 2006. Why central banks should not burst bubbles. International Finance 9: 109-24. [CrossRef]

Roubini, Nouriel. 2006. Why central banks should burst bubbles. International Finance 9: 87-107. [CrossRef]

Sargent, Thomas, and Neil Wallace. 1975. "Rational" expectations, the optimal monetary instrument, and the optimal money supply rule. Journal of Political Economy 83: 241-54. [CrossRef]

Shiller, Robert. 1981. Do stock prices move too much to be justified by subsequent changes in dividends? American Economic Review 71: 421-36.

Svensson, Lars. 2017. Cost-benefit analysis of leaning against the wind. Journal of Monetary Economics 90: 193-213. [CrossRef]

Wang, Jiang. 1993. A model of intertemporal asset prices under asymmetric information. Review of Economic Studies 60: $249-82$. [CrossRef]

White, William. 2009. Should monetary policy lean or clean: A reassessment. Central Banking 19: 32-42.

Yellen, Janet. 2010. Closing panel presentation. Journal of Money, Credit and Banking 42: 243-48. [CrossRef] 